stât, stâ nicht der mundart des dichters, sondern der literarischen tradition angehöre. Da ist nun aber nicht einzusehen, warum der dichter gerade im indicativ von gân bei seiner mundart geblieben sein, im indicativ von stân die mundartliche form ausnahmslos verbannt haben soll.

2. In der Kudrun reimt $\hat{\imath}$ auf ou 1 mal vor $m$, öfters vor $w$, vgl. Panzer s. 6 . Panzer hält s. 19 diese reime für unrein. Kaum mit recht. Vor $m$ ist der zusammenfall beider laute im bair.-östr. zwar nicht allgemein, aber weit verbreitet; vgl. Schmeller, Die mundarten Bayerns $\S \S 159.171$, Nagl, Vocalismus der bair.-östr. mundart s. 109, Pfalz, Die mundart des Marchfeldes (nr. 27 der berichte der phonogramm-archivs-kommission der kais. akademie der wissenschaften in Wien) $\S 17 \mathrm{c}, \S 20 \mathrm{c}$. Auch die reime vor $w$ sind rein.

WIEN.

M. H. JELLINEK.

\title{
AHD. ANTERÔN.
}

anterôn wird immer wieder mit 'ander' zusammengebracht, ohne daß man es recht glauben will. Der sinneszusammenhang ist keineswegs selbstverständlich, denn das wort bedeutet eben nicht 'ändern', auch nicht 'sich ändern, sich wie ein anderer gebärden', sondern transitiv 'den und den nachahmen'. 'ändern' dagegen heißt ahd. anderlîchôn.

Lautlich betrachtet müßte anterôn - es hat starres ahd. $t$ - in grammatischem wechsel zu 'ander' stehen, wie er wohl in ags. andergylde : óder vorliegt. Aber silbenanlautendes $t$ nach $n$ müßte ja im späteren althochdentsch wieder zu $d$ geschwächt sein, und der sorgfältigste beobachter dieser tatsache, Notker, hält, bei antroún das $t$ durchans fest. Damit erweist sich die anknïpfung an 'ander' als sicher falsch. desgleichen die übrigen bisherigen dentungsversuche?. 
Ich setze die beispiele aus Notker nach Pipers ausgabe rasch hierher:

'Terentius conticus tér nelêrta nieltt tie mores. unîlih sie unésen sïlîn.

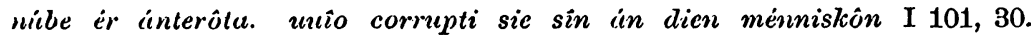
1)escripsi ... îh ánterôta 102, 1.

imitetur ... kécintrôt 236, 24.

Fóne diu cinterôt ter ciffo. dáz èr die ménnisken síhet tî̀on. Úbe ér iz in demo mûote êr gebíldôt nehábeti. so nemáhti er iz nîeht keánterốn I 343, 22-25.

imitatur ... ànterôn 351, 6.

Cumque non possit effingere eum. atque eqquare. Unde sô $s \hat{\imath}$ in geínterôn nemág. nóh síh imo geébenon 351, 8.

ęuulari ... keánterôn 351, 22.

Quid est gestus? 'Antpâra. tâtuuîchínga. ánterúnga. uuérbida 682, $15 \mathrm{D}$ (anterunga $H$; ânterûnga. anterunga $G$ ).

ystrionibus ... ánterârin 683,4 . $727,14$.

Petrógen gechôse dáz uuârheite gelîh ist. táz ánterot ten uuídellen

Tíz ist ter tóugeno cinafáng. tér in rhetorica héizet insinuatio. dér dáz ánterôt. táz mán in den bûosen slô̂fet 715, 7. $791,18$.

scis referre pindaream chelin. Dî chánst keánteron citharam pindavi

admixte musis. dedere etiam consonas gesticulationes. i. motus musicos. atque himeneia tripudia.

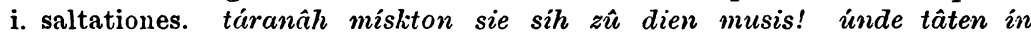
gerírte méttoda. dáz chît. sîe táten ín síngentên. mít íro líden. gehélle ánterungâ. iınde brûtliche trétenôda 803, 29.

Den ánteroton diê II 219, 5 .

tragedię ... An diên uuurden geántrôt fletus miserorum. nah demo innderskêite sexus et ętatis daz man fictis uocibus ketâte representationcm priami. alde hectoris. alde eccubę. alde andromachę... Vuanda diè ántrunga histriones tâten ora contorquendo. daz chit flannendo 639, 22-28.1)

Das wort findet sich naturgemäß gern in der sprache der kunst, der dramatischen zumal. Ich dachte eine zeitlang an fremde schauspieler und an das anklingende griech. anterān

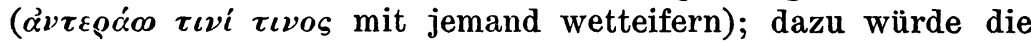
lautliche beobachtung passen, daß Notker das $t$ in einigen jungen lehnworten wie gemántelôte I 844, 11 nicht erweicht. Aber diese ganze brücke besteht doch nur aus luft.

1) Ich füge hier gleich einige zerstreute stellen aus sonstigen quellen ein: Emulatio antron plidon (lies pildon) Glossen 2, 733. Agit antrot 2, 417. $e z$... uuíl dìe ségela ántderôn Fundgr. I 21, 3. 
Nun gibt es ja auch andere -nt- bei Notker:

1. uuintere I 746,$25 ; 835,12$. uuinteres II 556,21. uuinterlichen I 746, 22. uuinterlichên 796, 31. $-t=t t=$ germ. $t$, verdoppelt durch $r$.

2. chunta II 146,$11 ; 147,9 ; 213,13$. chínton 407,1 . manta 119,$18 ; 407,15$. mánton 139, 21. nánton (zum infinitiv nenden) 357, 14. $-t=t t=$ ahd. $d+t$.

3. santa II 215, 16. hárasántost 21, 23. gesántiû 254, 23. suántin 51, 10. uuanta 274, 4. uuanton 321, 8. dánauuanti 317, 19. nezúnta 317, 20. inzuintiû 332, 7. $-t=t t=$ ahd. $t+t$.

4. Flectierte participia wie eruuindenta $\mathrm{I} 15,4$. ánasćhentin 15,26. vuîoffenten 17, $1 .-t=t t=$ germ. $d$, verdoppelt durch $j$.

Bei diesen vier gruppen hat die konsonantenverschärfung (trotz vorhergehender konsonanz) ihre spuren hinterlassen; das geschriebene $t$ ist ein verstärktes und fiel in der aussprache gar nicht hinter die silbengrenze, konnte also auch nicht geschwächt werden. Hierzu kommen nun noch die vielen fälle, wo auch der ungeschulte die silbengrenze merkt und keine erweichung erwartet:

5. geäntuúrten I 15, 10; cintpûra 682,15 u.s.w.

ánterôn gehört - zur fünften gruppe, wie das Keronische glossar erweist (Ahd. Glossen I 28). Die handschrift Pa hat aemulis antharonti, imitatur antharari, aenulo antharom, aemula antharota, imitatrix anthara, aemulatio antharunga ${ }^{1}$ ) Diese worte machten Kögel in seiner abhandlung über das Keronische glossar schwierigkeiten, da er sie weder bei ahd. $d$ noch bei ahd. $t$ recht unterbrachte (z. b. s. 115 f.). Aber jede schwierigkeit fällt, wenn man aint-harônti u.s.w. liest. Auch Notkers formen sind damit erklärt. Das $t$ konnte nicht geschwächt werden, weil es zur zeit der erweichung die silbe nicht eröffnete; $h$ in der fuge, zumal dunkler worte, ist stets gefährdet: das verblassen des mittleren vokals ist lautgesetzlich; auch die vereinzelten fälle von synkope lassen sich rechtfertigen (falls sie überhaupt dem verfasser und nicht den schreiber! zuzuweisen sind).

3) Die entsprechenden atellen der hs. $K$ sind: nemulus anftomd. innitatur antrari, emnlo antrom, aemulo antronda, inmitatris antrant. ęunulacio antrunga. 
Natiurlich darf man in dem wort keine zusammensetzung sehen, sondern eine ableitung mit dem ton auf der ersten silbe. 'Zu ahd. harên 'rufen' gab es, glaube ich, ein männliches cintharo, und davon ist cintharôn abgeleitet. Jenes * (intharo hängt keineswegs in der luft. Das femininum anthara habe ich eben aus $\mathrm{Pa}$ angeführt, das masculinum antharari (später cinterciri antrer) ist eine neubildung wie 'geber' für älteres gebo; in den Murbacher Hymnen XXV 2, 1 steht noch ein foraharo 'praeco'1). In laut und bedeutung bald verändert behielt cinterôn die urdeutsche betonung und ragt als der seltsamste rest der alten vorsilbe aint- neben Antlitz, Antwort, anheischig in das heutige deutsch bez. dessen mundarten hinein.

Verbale zusammensetzung ist dagegen ags. onhyrian 'nachahmen'. Es verhält sich zu ahd. antharo(n) ungefähr wie ahd. intsizzan : ántsâzzic, ags. onfôn : andfenga. BosworthToller bietet s. 755 zahlreiche belege für onhyrian mit den ableitungen onhyriend m., onhyring und onhyreness f. Auch einfaches hyrian scheint vorzukommen, King Alfred's Boethius (Sedgefield) s. 108, 11 und 146, 7, doch beidemal durch paralleles on- gefährdet. Endlich findet sich cefterhyrigean, das bei Bosw. s. 11 fälschlich den akut trägt (richtig in Early English Text Society 110 f., s. 226). Mit hýran 'hören' hat das wort nichts zu tun, obwohl es die Bedaübersetzung einmal damit zusammenbringt (EETS. 95 f. s. 2, zeile 8): se de hit gehyrep, he onhyrep ham. Es hat kurzes, echtes $y$ (= kent.e), geht nach der ersten schwachen conjugation und steht zum ahd. im ablaut wie ahd. gripfen : greifôn (: grîfan). Die erste hochstufe hierzu liefert ahd. herêta 'ich rief' im dritten bruchstück des alem. psalters (Braunes Lesebuch ' s. 41). Angesichts dessen darf auch hérenten erwähnt werden, bei Notker II 62,10; dieser selbst wird, wie Piper vorschlägt, das gewöhnliche hárenten geschrieben haben, hérenten aber kann ein Alemanne gesprochen haben, der Notkers psalmen abschrieb.

1) Vielleicht gehört der eigennamen Andahar hierher (Förstemann" I 103), sowie die ortsmamen Antheringa II 148, Anteresbach 149. Überhaupt ist es eine lockende, aber doch wohl trügerische aussicht, eigennamen auf -har-, die mit der bedeutung 'heer' keinen sinn geben, zu übersetzen als 'rufer'. 
Die vocalstufe von ahd. antharôn selbst liegt noch vor in an. herma < harmjan. Das $m$ wird aufzufassen sein wie in nhd. schwärmen : schwirren, ahd. galm : galan, weist jedenfalls auf ein substantivum harm. ${ }^{1}$ ) Einfaches herma bedeutet '(wieder) berichten', dagegen herma eptir (mit dativ) = 'nachalımen'. Hierzu eptir-hermur f. pl. 'das nachmachen von stimme und gebärde', hermi-kv'áka f. 'spottvogel' (Cleasby-Vigfusson s. 131 u. 258).

Die außerdeutschen beispiele räumen zugleich bedeutungsschwierigkeiten hinweg. Im ahd. kommt man mit der allgemeinen bedeutung 'jemand darstellen, agieren' aus, man müßte denn das beispiel Notker II 639 pressen. Das Schweizer Idiotikon I 349 betont für die lebenden mundarten das 'nachmachen mit der stimme' schon etwas mehr, und für das altn. ist der zusammenhang von 'rufen' und 'nachmachen' offenbar. Im aengl. spürt man den sinnlichen inhalt am ehesten in den Exeterrätseln 9 u. 25:

ic... sceávendvîsan hlûde onhyrge (Grein 2, s. 374), und besonders

Ic eom vunderlicu viht, hvîlum beorce svâ hund, hvîlum groede svâ gôs, hvîlum ic onhyrge gû̃fugles hleóðor, mû̀e gemcene, boer ic glado sitte. vrosne mine stefne:

hvîlum Ulcete svâ gât, hvîlum gielle svâ hafoc, pone hasvan earn, hvîlum glidan reorde hvîlum maves song,

(Grein 2, s. 381).

Dieses rätsel meint den 'häher'; und merkwürdig, noch Konrad von Megenberg sagt in seinem deutschen Buch der Natur (Pfeiffer s. 199, 12) vom häher: er äntert (d. l. änteret $A$, endert $B d$, antwort a) all ander vogel mit der stimm, alsó daz er sein stinm anderr vogel stimm geleicht, reht sam or ir spot. Und hierzu paßt wieder an. hermi-kráka.

Die bedeutungsentwicklung von antharon ist also: als gegenredner jemand anrufen ${ }^{2}$ ) - spöttisch jemand nachmachen;

') Vielleicht hängt dieses harm mit dem gleichlantenden weitverbreiteten wort zusammen. Dieses hat in den alten denkmälern vielfach den sinn 'beleidigung' und künnte von unserem voransgesetzten wort '(spottender) zuruf' minlestens beeinfluBt, sein.

2) Das ahd. wort ist transitiv, etwa wie foralotion 'vorherverkinden: Das sprachgefihl der gegenwart erwartet eher den dativ. Dieser iilure wiegt bei ags. onhlyriun. 
darstellen mit der stimme - darstellen schlechthin. Wie rasch eines ins andere übergeht, zeigt am besten das Harbardoslied :

\author{
pórr kallapi: \\ ' Huerr er sá sueinn sueina, \\ er stendr fyr sundit handan?' \\ Hann suarabi: \\ 'Huerr er sí karl karla, \\ er kallar um uáginn?'
}

Man kennt die streitlust der alten Germanen, ihre freude an gegenrede, einwurf, widerwort. Dergleichen übten sie vor dem zweikampf in der schlacht, sie liebten es innerhalb des heldengedichtes, sie hatten eigene spottgesänge, weisheitswettkämpfe, süngerstreite. Ich glaube, in dieser mehr dramatischen dichtung war nicht nur das zeitwort antharôn, sondern auch das substantiv *antharo fester fachausdruck, es kam männern zu wie Unferð und vielleicht auch Hadubrand.

Wie man ags. wóp, an. ódr 'gesang' ins indogermanische zurückverfolgt und ein zeugnis für uralte poesie darin sieht, so ist ahd. antharôn und seine sippe ein stehender ausdruck der germanischen dichtung und hat anderwärts entsprechungen. Das einfache harên stellt sich am besten zum reichentwickelten stamm hrô-, griech. xrí es, wie bei einem entfernteren, aber doch verwandten wort dem antharôn ein gegenstück ersteht: aemulationes anthruoft (Braunes Lesebuch ${ }^{7}$ s. 37, 40). bleiben.

Dagegen müssen got. hazjan, ags. herian 'preisen' fern-

FREIBURG i. B., 18. Juli 1914.

ERNST OCHS. 